\title{
Algunas influencias en la autonomía de los periodistas chilenos: una actualización de investigaciones previas
}

\section{Some influences on the autonomy of Chilean journalists: an update of previous research}

\section{Francisca Greene González ${ }^{1}$}

Recibido: 20-03-2017 - Aceptado: 19-07-2017

DOI: https://doi.org/10.26441/RC16.2-2017-A11

RESUMEN: Entre 2002 y 2012 se realizaron algunas investigaciones que concluyeron que los periodistas chilenos carecían de independencia y autonomía al realizar su trabajo. En ellas, se constató que había influencias de sus editores, además de las presiones ejercidas por la empresa y los auspiciadores.

El presente trabajo tiene como objetivo -a 15 años del comienzo de esas investigacionesanalizar algunas de las influencias de los periodistas. Se pretende evaluar las conclusiones de las investigaciones previas y agregar otro factor: el efecto de las redes sociales como nuevo elemento de presión.

Se hicieron entrevistas en profundidad a ocho periodistas que ejercen como reporteros en distintos medios de comunicación nacionales. Los periodistas elegidos fueron jóvenes, precisamente para evaluar nuevas opiniones en cuanto a la autonomía percibida.

Se concluyó que, para la mitad de los periodistas entrevistados, las presiones económicas de los dueños de la empresa les resta autonomía; la mayoría no se siente presionado por su editor y las redes sociales significan, para la mitad de los entrevistados, un factor para tener en cuenta en sus publicaciones.

Palabras clave: periodistas; presiones laborales; autonomía; editores; empresa periodística.

ABSTRACT: Between 2002 and 2012 some investigations were carried out that concluded that Chilean journalists lacked independence and autonomy when carrying out their work. In them, it was found that there were influences of its editors, in addition to the pressures exerted by the company and the sponsors.

The aim of this paper is to analyze some of the influences of journalists, 15 years after the beginning of this research. It is intended to evaluate the conclusions of previous research and add another factor: the effect of social networks as a new element of pressure.

In-depth interviews were conducted with eight journalists who worked as reporters on various national

1 Francisca Greene González es Periodista por la Pontificia Universidad Católica de Chile, Doctora en Ciencias de la Información por la Universidad Complutense de Madrid, España, Profesora de Fundamentos de la Comunicación y Ética Periodística de la Facultad de Comunicación de la Universidad de los Andes, Santiago, Chile. fgreene@uandes.cl, http://orcid.org/0000-0002-0538-2584. 
media. The elected journalists were young, precisely to evaluate new opinions regarding perceived autonomy.

It was concluded that, for half of the interviewed journalists, the economic pressures of the owners of the company lack autonomy; Most do not feel pressured by their editors and social networks mean, for half the interviewees, a factor to take into account in their publications.

Keywords: journalists; labor pressures; autonomy; editors; journalistic company.

\section{Introducción}

La autonomía de los periodistas ha sido un tema largamente estudiado bajo diferentes perspectivas. Aunque a veces se ha cuestionado la neutralidad, producto principal de la autonomía, como una norma exigible a los periodistas (McDevitt, 2003; Schudson, 2005), la mayoría de los investigadores coinciden en que, en una democracia, el interés público es el criterio que debe usarse para hacer juicios noticiosos que garanticen la neutralidad. Los autores definen el interés público como elemento de control a las instituciones, informar a los ciudadanos y/o permitir un cambio social positivo (Hallin y Mancini, 2004; McQuail, 1992). Sin embargo, aunque parezca incoherente, asegurar la autonomía sobre la toma de decisiones editorial ha llegado a ser más difícil para los periodistas después del auge de las democracias a partir de 1973 (Dunham et al., 2015; Mair, 2011).

Nygren (2012) sitúa la autonomía profesional de los periodistas en dos niveles. El primer nivel es la autonomía del periodista que trabaja en la organización o lo que Nygren (2012) llama "autonomía interna" o "autonomía de los periodistas en su trabajo diario en relación con el medio". El segundo nivel, “autonomía externa”, es la autono- mía de la profesión como grupo en relación con los actores y las ideas en la sociedad. Sjovaag (2013) desarrolla aún más este argumento, aclarando que la autonomía

...está restringida en los niveles político, económico y organizativo de la producción de noticias, negociada a nivel editorial y ejercida a nivel práctico”. Así, la autonomía es "un concepto fluido que se ajusta continuamente para gestionar la tarea diaria de informar las noticias" (Sjovaag, 2013).

En los estudios empíricos, la autonomía profesional se ha operacionalizado como un juicio editorial dentro del medio, es decir, la autonomía "interna" de Nygren (Mellado y Humanes, 2012; Skovsgaard, 2014; Weaver et al., 2006; Weaver y Willant, 2012). La autonomía externa no se ha medido directamente en encuestas a periodistas. En su lugar, las presiones extra-media se han operacionalizado ocasionalmente como posibles influencias sobre la autonomía. Weaver et al. (2006) analizaron las variables demográficas y ocupacionales individuales, las características de la organización de noticias y las percepciones de las metas y prácticas organizacionales como predictores de la autonomía percibida. 
Encuestas en 18 países políticamente diversos examinaron las influencias sobre la autonomía y el trabajo periodístico. Más allá de las rutinas periodísticas, los investigadores identificaron influencias percibidas correspondientes a seis dominios (Hanitzsch et al., 2011; Hanitzsch y Mellado, 2011). Reich y Hanitzsch (2013) encontraron asociaciones entre las influencias percibidas, las influencias políticas, organizativas, de procedimientos y profesionales. El tipo de propiedad de los medios de comunicación, rango organizacional y años de experiencia fueron los predictores objetivos más fuertes. El desempeño democrático nacional también fue un fuerte predictor, los países con democracias consolidadas tienen periodistas con mayor sensación de autonomía.

Los últimos estudios latinoamericanos sobre autonomía periodística analizan las restricciones que impone el poder político en Argentina y la escasa disponibilidad de fuentes en tiempos de elecciones (Amado, 2015). Amado (2010) hace un interesante análisis de las presiones ejercidas sobre los periodistas por los objetivos personales, institucionales y sociales.

En Colombia y México se estudió la presión ejercida por los editores y la influencia de regímenes donde no está garantizada la democracia para definir autonomía profesional (Barrios and Arroyave, 2007; Guerrero y Márquez-
Ramírez, 2014). En estos países donde hay fuerte control político, los estudios concluyen que la autocensura no es el enemigo más peligroso de la libertad de expresión sino la censura generada por la intimidación, las amenazas y los asesinatos, cuyo fin es silenciar el trabajo (Gutiérrez y Torres, 2014) .

Sin embargo, Ortega y Humanes (2000) concluyen que existe un amplio margen para actuar dentro de los medios, como si éstos sólo marcasen débiles constricciones a cambio de poner a disposición de los profesionales los medios necesarios para el ejercicio de su labor. Así, el poder de los medios de comunicación tiene su mayor limitación en el poder de sus periodistas.

Un estudio financiado por la fundación Konrad-Adenauer ${ }^{2}$ en 2016 muestra que, en general, los periodistas latinoamericanos manifiestan tener una gran autonomía para realizar su trabajo. Tres profesionales de cada cinco dicen tener total o gran libertad para elegir las historias que reportean $(67,9 \%)$. $\mathrm{Al}$ analizar los datos por tipo de medio, no se perciben grandes diferencias en la libertad percibida en medios privados, estatales o de la sociedad civil (públicos, en el encuadre de la encuesta). Contrariamente a lo que sostuvo parte del debate entre gobiernos contra medios y periodistas, que acusaban de manipulación a los empresarios en la actividad periodística, los periodistas de medios privados son los que expresan

2 Amado, Adriana (editora). El periodismo por los periodistas Perfiles profesionales en las democracias de América Latina. Recuperado de http://www.kas.de/wf/doc/kas_48177-1522-4-30.pdf?170310150429. 
mayores niveles de autonomía. Percepción similar se registró con relación a la libertad para decidir qué aspectos enfatizar de la noticia $(68,9 \%)$, aunque solo la mitad reconocen participar en las reuniones editoriales $(52,8 \%)$. Nuevamente el porcentaje levemente más alto asignado a "ninguna o poca libertad" en la selección de los temas se registra en los medios estatales, que permiten un mayor grado de libertad a la hora de elegir el enfoque. La percepción de autonomía es alta en países con distintos niveles de libertad para la profesión. Países con la situación más crítica, motivada por agresiones graves $\mathrm{o}$ asesinatos a periodistas, como México, Venezuela y Colombia, ocupan las últimas posiciones hacia 2015. Ecuador y Brasil muestran un agravamiento de la situación que los corre por encima del puesto 100 desde 2011. En cambio, Argentina, El Salvador y Chile parecen haber mantenido más o menos cierta estabilidad según esa medición.

En Chile se han realizado numerosos estudios sobre autonomía periodística. Gronemeyer (2002) concluye que, además de tener en cuenta que su trabajo es para los lectores, el periodista debe adaptarse a las políticas del propio medio, pero sin dejar de desarrollar espacios de autonomía e independencia que se hacen exigibles para cumplir con el fin del trabajo periodístico. Greene (2012) agrega que las redacciones necesitan autonomía y libertad externa e interna para cumplir adecuadamente con el deber de informar y, cuando este clima no existe, tampoco puede existir la objetividad. Lecaros (2004) añade que la existencia de libertad es condición fundamental para que los periodistas puedan realizar a cabalidad su deber de informar, deber que se sustenta en el derecho del público a ser informado y que, por tanto, constituye un acto de justicia. Lecaros introduce un nuevo factor, el de la justicia, indicando que el periodista debe informar con absoluta libertad y autonomía porque es lo justo para él y su desarrollo como profesional y también es lo justo para la sociedad que demanda y exige la información objetiva.

Este estudio pretende observar, a diez años de tres estudios importantes sobre autonomía periodística en Chile, si las presiones observadas por los periodistas en ese entonces continúan. Se ha agregado otro factor que tiene relación con las posibles presiones introducidas con las nuevas tecnologías. En los tres estudios anteriores se habían considerado dos grandes factores: el papel del editor y el de la empresa periodística. Se trata de los estudios de Yez (2011), Gronemeyer (2002) y Mellado (2012).

Hoy es imposible desconocer que las nuevas tecnologías han dado un poder casi ilimitado al público, lo que condiciona el trabajo periodístico. Al mismo tiempo que se desarrollan las redes sociales, la academia reflexiona en torno a la influencia que éstas tienen en el trabajo de las salas de redacción de los medios. Los estudios en este campo son numerosos: Domingo et al. (2007), Orihuela (2008), Flores Vivar (2009). Campos Freire (2008) concluye que el 
rápido éxito de las redes sociales ha encendido las alarmas en los medios de comunicación tradicionales. Señala que el fenómeno de las redes sociales suma audiencias millonarias, incrementa su publicidad, logra la personalización de los usuarios y rompe con algunas de las barreras de las viejas organizaciones mediáticas.

Además de las redes sociales, la tecnología ha abierto diferentes puertas al periodismo, que los medios han aprovechado disponiendo nuevas plataformas al público. Los usuarios pueden mandar fotos y videos, incluso pueden proponer temas de investigación. Por lo mismo, los contenidos generados por los usuarios son cada vez mayores (Greene, 2014).

Estudios ingleses han concluido que la creación del contenido ha dejado de ser exclusiva de una élite de periodistas y que la participación pública en las pautas y los propios contenidos es creciente (Blank, 2013; Dutton \& Blank, 2013). Un estudio de caso en Inglaterra, que incluyó encuestas a ciudadanos y periodistas, introdujo el concepto de "quinto poder" que se atribuye a la capacidad del público de generar sus propios contenidos en la red. Estos contenidos son recogidos por el tradicional "cuarto poder" dando origen a una nueva ecología de las noticias (Blank, Dutton, Newman, 2012).

\subsection{El papel del editor}

Sancho (2004) destaca varias virtudes indispensables del editor: debe ser un líder, debe ser escuchado y admirado por sus periodistas, debe ser capaz de guiar a un equipo y sacarle lo mejor de sí. Así, dice, no se debe confundir el "mandar" con el "liderar".

Lecaros (2009) añade que "anticipar los problemas pasa a ser una exigencia ética. El editor debe pensar antes de entrar en la convergencia”. La autora ofrece un listado de cuestiones sobre las cuales los editores deberían deliberar, por ejemplo, si hay que unificar o no estándares y rutinas en la obtención de la información, en la producción y en la emisión de ella. Otras atribuciones definidas por Lecaros son: anticiparse a entender la sensación del periodista que siempre pensó ser "propietario" de la noticia aunque nunca lo haya sido en términos reales; definir cuáles serán los criterios de verificación; determinar cuándo un mismo material "se le debe" a un canal de televisión y a una página web; saber cómo se determinará el "valor noticioso" de un hecho cuando éste debe ser compartido, producido para medios impresos, televisivos y online; cómo se corrige una información en las multiplataformas y, así, un listado largo de cuestiones que refiere a los nuevos desafíos impuestos a los editores al disponer de plataformas al público para el comentario, la opinión y la denuncia.

Las habilidades y conocimientos técnicos del editor se adquieren durante mucho tiempo, señala Giles (1993). Con los años, el reportero demuestra un comportamiento que atrae la atención de los grandes editores: buenas ideas, un fuerte sentido de lo que significa la noticia, gran motivación, compromiso con el trabajo del diario, productividad. En la naturaleza del 
negocio de las noticias, los individuos con estas cualidades son quienes son "ascendidos al escritorio", ellos llegan a ser supervisores con la responsabilidad de administrar a otros y producir resultados.

El estudio de Puente, Edwards y Delpiano (2014) concluye que, si bien las funciones del periodista y del editor no se han modificado, no ha ocurrido lo mismo con sus responsabilidades: antes bastaba con elegir las noticias más interesantes para el medio, mientras que hoy, más que nunca, es preciso perfeccionar la selección y jerarquizar su valor social. El estudio destaca que es imprescindible dar contexto a las noticias, definir un foco propio del medio y aumentar el número de fuentes.

\subsection{La empresa periodística}

No tendría sentido exigirle a los comunicadores que sean responsables con la sociedad si el medio no dispusiera de unas orientaciones que le facilitaran cumplir con sus propios compromisos con la comunidad.

Sánchez Tabernero (2000) aclara que, cuando se habla de que la empresa debe cumplir con un código ético, no se debe pensar en limitaciones que coartan la libertad o autonomía del periodista. Y cuando se habla de que la empresa debe ser coherente con lo que pide es para que los trabajadores compartan determinados valores.

Un estudio de Taufic (2005) concluye que, para que la empresa cumpla con su rol de promotor de la autonomía e independencia de sus periodistas, debe entregarles las herramientas pertinentes; debe dejar en claro su línea editorial para que así los comunicadores entiendan con claridad cuáles son sus parámetros editoriales. Para mantener el rumbo, los grandes medios y organizaciones de comunicación de muy distintos países se han dotado, desde los inicios del siglo XX, de manuales de estilo, primero, y luego de códigos de ética periodística que obligan a sus propietarios y redactores ante la opinión pública y sus lectores a un determinado comportamiento editorial, que todos conocen y pueden exigir.

El estudio señala que en Chile hay falencias en el tema ya que, si bien los directivos necesitan plantillas competentes y motivadas en las que la persona se plantee cómo mejorar su trabajo y cómo contribuir al mejoramiento de su empresa, dicha información (códigos de ética) debiese ser de carácter público para todos los usuarios de aquel medio.

Después de una encuesta aplicada a un número importante de periodistas, Taufic (2004) concluye que el mejor periodismo se rige por deberes autoimpuestos que, además de dictar las reglas del juego para sus periodistas, el marco y límites del propio medio, deja en evidencia un compromiso explícito con la sociedad y opinión pública, en cuanto a veracidad e imparcialidad.

Mercedes Muñoz (2004) coincide con Taufic y considera indispensable para la profesión periodística formar en la autorregulación. Sostiene que debe existir una serie de prácticas que informen la actividad periodística y 
que, paulatinamente, constituyan hábitos que capaciten a los profesionales para escoger la solución más ética, que no es otra que la más profesional, en el conflicto más difícil.

Aznar (2004) aclara que un buen uso de los códigos no lo es todo. Ellos no pueden evitar -ni es su función- la necesidad de que el profesional reflexione sobre las circunstancias de cada situación en la que se halle; ni pretenden evitar que los profesionales se planteen cuestiones de más alcance acerca de su papel y el de los medios en la sociedad actual. Pero lo que sí hacen los códigos es facilitar las pautas normativas que el profesional debe aplicar a estos casos y que le sirven por tanto de guía a su práctica diaria.

Estar en comunión con la misión de la empresa es algo que, si bien no es condición para trabajar en un medio, trae consecuencias convenientes para el periodista. Cuando la misión es compartida por quienes trabajan en las empresas, "éstas pueden sobrevivir a sus promotores; y los cambios en la cúpula de la organización no suponen cataclismos sino un nuevo impulso para la empresa" (Sánchez Tabernero, 2000).

Lecaros (2004) agrega que la autonomía interna, la libertad que cada profesional tiene para actuar, es una libertad que no es "ganada" por la profesión en una acción reivindicacionista y monopólica, como tampoco es otorgada a un profesional determinado en razón de su acierto en la acción. La libertad que tienen los periodistas al interior de la propia profesión es exigida por la sociedad. El público no quiere una receta fija, un modo único de acción por parte de los profesionales: quiere, cada vez, la mejor de las acciones posibles, y es por esto que exige para cada periodista esa libertad.

La libertad de expresión y el derecho a la información no son sólo amenazados por la autoridad o por legislaciones rígidas; también ha influido la globalización, la concentración de la propiedad de los medios de comunicación, el influjo de sus avisadores y el choque de intereses de la gerencia. El informador no es inmune a todo lo planteado, pero su medio tiene que cumplir con su trascendente rol social en medio de un fuerte juego de presiones cruzadas (Taufic, 2005).

\subsection{Investigaciones y conclusiones previas en Chile}

En 2011 Lyuba Yez estudió las fortalezas y debilidades existentes en el periodismo chileno a partir de una investigación basada en una muestra de 300 individuos que ocupaban algún cargo en un medio informativo en la capital. Dentro del grupo de personas había reporteros, editores y ejecutivos de la empresa.

Dentro de las conclusiones principales del estudio de Yez, se encontraron interesantes relaciones entre la autonomía profesional y las presiones externas a las cuales el periodista se veía sometido.

Según la investigación, la guerra por el rating y la preocupación por los resultados económicos debilita el papel del periodista: las metas económicas 
presionan y, a la vez, dañan la calidad del ejercicio periodístico. El 47,3\% de los encuestados aseguró que esa es la principal preocupación del departamento de prensa.

Otro tema importante del estudio es la actitud del periodista al momento de cubrir una historia en la que se involucren o afecten los intereses de los dueños del medio en el que trabaja. Yez señala que existe más desconfianza $(55,7 \%)$ que confianza $(42,1 \%)$ respecto de su independencia en la cobertura de noticias que afectan los intereses corporativos de los dueños del medio.

$\mathrm{Y}$ en cuanto al sentimiento de autonomía, más de la mitad de los encuestados por Yez aseguró que no ha sido motivado a cubrir historias relacionadas con los dueños o auspiciadores de su medio.

El estudio concluyó que respecto a la "convivencia entre lo económico y lo editorial", se plantean inconformidades e incomodidad para los periodistas, lo que afecta la cobertura de ciertas noticias.

María Elena Gronemeyer desarrolló en 2002 una investigación que constató, mediante una encuesta nacional aplicada a periodistas y editores, además de entrevistas y conversaciones en grupo, que existía una "grave dosis de inseguridad y conformismo" en cuanto a la autonomía e independencia de los periodistas chilenos.

Su estudio se basa en los resultados de una encuesta realizada en 2001 a nivel nacional donde se buscaba medir la confiablidad de 15 profesiones. Los periodistas resultaron ser los profesiona- les menos confiables de la sociedad según las conclusiones de dicho estudio.

Gronemeyer explica que, desde que Chile volvió a la democracia en 1990, los periodistas excusan sus debilidades atribuyéndolas a la experiencia de censura durante el régimen militar entre 1973 y 1989 y al sistema de libre mercado imperante hoy en día que influye en las pautas de las empresas periodísticas.

Pero la investigación no critica sólo a periodistas, sino que también hace alusión a las falencias de los editores respecto de su papel en el refuerzo de la seguridad de quienes están bajo su mando y a la presión que pueden ejercer incorrectamente sobre ellos, generando inestabilidad. Según los mismos implicados, editores y periodistas, el bajo nivel de compromiso con la autonomía e independencia se debe "a un espíritu conformista y a un grado importante de inseguridad" (Gronemeyer, 2002).

El estudio explica que, si bien en una situación ideal de conflicto entre la empresa y el periodista se debiera tratar de manejar las diferencias y lograr una dinámica de consenso y entendimiento, en la realidad se opta por evadir las polaridades; y es en dicho intento de eludirlas que "editores y periodistas tienden con frecuencia a someterse a las exigencias impuestas por la empresa aun sacrificando exigencias éticas de su profesión y claudicando a sus espacios de autonomía e independencia" (Gronemeyer, 2002).

El estudio determinó que los periodistas percibían a dueños y directivos 
de su medio de comunicación y a la gerencia en general, como individuos interesados en fines muy diferentes a los propios, con un uso de valores diferente y metas no convergentes.

Una de las consecuencias más graves para Gronemeyer es que la falta de ambos valores -autonomía e independencia- conlleva a una actitud de dependencia y sumisión. Una de las repercusiones de esto es que la sociedad se ve directamente perturbada y se deja el camino libre a quienes sustentan el poder político y económico, para que definan la agenda noticiosa en beneficio propio, sin consideración del bien común o incluso en contra de éste.

Claudia Mellado (2011) realizó un estudio basado en 570 encuestas a periodistas chilenos. Su objetivo fue percibir el nivel de autonomía que tenían los comunicadores en su trabajo cotidiano, la importancia que le daban a dicho valor y divisar cuán libres se sentían frente a la línea editorial.

En la investigación, Mellado (2011) postula que dentro de un mercado de trabajo irregular, con empleos flexibles y de corto plazo y en el cual hay una imposición de la tecnología y una disminución de la clasificación, se produce un empobrecimiento del compromiso de los periodistas con sus valores profesionales, entre ellos, la autonomía.

El estudio destaca que los periodistas con más experiencia tienen más poder y el poder otorga independencia y seguridad en la sala de prensa. Por lo tanto, los periodistas más antiguos sienten una mayor libertad en su trabajo.

Mellado recalca además que, si bien
Chile está entre los países catalogados que tienen prensa libre, clasificados así por encima de otros países latinoamericanos según el Ranking de Freedom House 2011, hay varios factores que aún sugieren una falta de libertad en el trabajo diario del periodista chileno. Según la autora, hay diversas investigaciones que atribuyen la situación a la censura del periodo de gobierno militar, además de una baja calidad de enseñanza en las universidades y la mala imagen que tiene el periodista frente a la sociedad.

Mellado aplicó una encuesta vía internet. La lista de encuestados incluyó periodistas, escritores, comentaristas de noticias, columnistas, correctores, redactores, editores, presentadores y los productores de noticias que trabajaban en distintos medios como agencias de noticias e Internet. El cuestionario trató cuatro temas relacionados con la autonomía.

Uno de los objetivos de la investigación fue determinar si la autonomía era considerada un valor importante entre los periodistas. Los datos revelaron que los encuestados consideraron dicho principio como el quinto factor más valorado, siendo superado por factores relacionados con condiciones laborales. Mellado explica que esto se debe al subdesarrollo que tiene el periodismo como profesión en Chile y que las malas condiciones de trabajo están al descubierto, como por ejemplo, los bajos sueldos, la inestabilidad, los horarios extendidos, entre otros. La autonomía, por lo tanto, cumple con ser un elemento muy importante para los periodistas 
pero no sustituye a las necesidades materiales de los comunicadores.

Mellado (2011) explica que, en un mercado altamente concentrado como el de Chile, donde los medios de comunicación están en manos de la elite político-económica y aún hay leyes que restringen la libertad de prensa, es difícil que los periodistas sean completamente libres.

Los resultados de la investigación demuestran que los periodistas encuestados se sentían cómodos y libres frente a la toma de decisiones sobre la pauta. Respecto de su autonomía explicaron que ésta la relacionaban con dos concepciones principales: servicio al público de manera correcta y su desarrollo profesional y laboral. Además se concluyó que los factores económicos no tienen ningún efecto directo sobre dichos profesionales, pero que, en contraste con lo anterior, se admite que se sienten influencias en los aspectos políticos y por parte de la organización y la empresa.

\section{Metodología}

La motivación por realizar este trabajo nació a partir de las investigaciones previas de las autoras descritas anteriormente que concluyeron que los periodistas chilenos carecen de dos valores fundamentales para su desarrollo profesional: la autonomía y la independencia.

Ante la falta de nueva evidencia que retrate el grado de autonomía de los periodistas chilenos hoy, esta investigación se centró en las inquietudes constatadas en estudios pasados y en pre- guntas nuevas relacionadas con la presión ejercida por el público a través de redes sociales y otros mecanismos de opinión, para determinar cuáles son los temores del periodista y los factores que restringen la libertad en el desarrollo de su trabajo.

La investigación se basó en entrevistas en profundidad a ocho periodistas que cumplían con las siguientes condiciones: (a) estar actualmente trabajando en un medio de comunicación y (b) no llevar más de cinco años en ejercicio de la profesión. Esto, debido a que está comprobado por los estudios previos que, cuando un periodista lleva más tiempo en el medio, está más seguro de sí mismo y de su puesto de trabajo. Se entrevistó a tres periodistas que trabajaban en el departamento de prensa de tres canales de televisión diferentes (TVN, Canal 13 y Chilevisión); dos que se desarrollan en prensa escrita $(E l$ Mercurio y La Tercera); uno que ejerce como periodista de radio (Bío Bío); uno que trabaja en una revista (Qué Pasa) y, por último, uno que trabaja en una página web de noticias (Emol). Se eligieron estos medios porque se trata de los medios con mayor rating en el país. Pareció importante la diversidad de soportes para analizar si las percepciones sobre el grado de autonomía tenía relación con las características del medio.

Las preguntas fueron formuladas con la idea de poder sacar algunas conclusiones acerca de las influencias externas que reciben los periodistas (políticas y económicas), la percepción de éstos en cuanto a sus editores y los 
dueños de su medio de comunicación y las posibles presiones de parte del público.

Las entrevistas fueron hechas de modo presencial para así poder lograr conversaciones donde los entrevistados no se sintieran limitados y pudiesen aportar la mayor cantidad de información posible.

Esta investigación se desarrolló durante abril de 2016. Se trató de un trabajo exploratorio (Hernández, 2003), pues provee datos que pueden ser clasificados, ordenados, analizados e interpretados para descubrir ideas y relaciones nuevas (Vieytes, 2004). Además, fue descriptivo (Hernández, 2003), para así predecir -aunque sea en un grado mínimo- el comportamiento del objeto (Vieytes, 2004).

Según el método utilizado, fue cualitativo, orientada a captar el origen, el proceso y la naturaleza de los significados de los actos de los sujetos investigados, que brotan de la interacción (Ruiz Olabuénaga, 2003). Su intención fue entender los acontecimientos, acciones, normas y valores desde la perspectiva de los propios sujetos que los producen y experimentan, preguntándose qué piensan, cómo viven o experimentan un cambio en sus hábitos y costumbres (Vieytes, 2004). Se seleccionaron para las entrevistas a miembros representativos de los medios nacionales que han adoptado las nuevas tecnologías en su modo de trabajo diario.

Se aplicaron entrevistas del tipo $s e-$ miestructurada, basadas en una guía de preguntas y asuntos específicos (Hernández, 2003), también llamada entrevista estandarizada abierta (Canales, 2006). Las entrevistas duraron aproximadamente una hora y media en donde se realizó un cuestionario con quince preguntas. La entrevista en profundidad se ha validado como un método de levantamiento de material de estudio sustentado en el valor atribuible a respuestas honestas y significativas en una conversación cómoda y sin esquemas fijos o categorías de respuestas predefinidas (Ruiz Olabuénaga, 2003; Guerrero, 2014).

\section{Resultados}

Los entrevistados fueron:

3 periodistas de Televisión: Caso 1, Caso 3, Caso 7.

2 periodistas de Prensa Escrita: Caso 4, Caso 6.

1 Periodista de Radio: Caso 2.

1 Periodista de Revista: Caso 8.

1 Periodista de un medio digital: Caso 5.

Las respuestas entregadas por los entrevistados fueron divididas en los siguientes subtemas: presiones económicas, presiones políticas, presiones personales, presiones de autoridad y presiones de la opinión pública. Se transcriben extractos de las respuestas más releventes.

\subsection{Presiones Económicas}

Se preguntó a los periodistas si sentían alguna restricción de parte de la empresa, pues muchas veces el dueño del medio es propietario de otras empresas, lo que provoca conflictos de intereses. El interés de la gerencia por obtener utilidades puede no coincidir con las orientaciones programáticas de 
un medio. Según los entrevistados, el periodista tiende a velar por la calidad de las noticias, mientras que los dueños, por el rating.

Caso 1: "Me parece que el conflicto principal en este sentido es la búsqueda de rating por sobre la calidad de la información. Muchas veces, se reportean temas buscando "la venta" más que otros factores que pueden ser más importantes".

Caso 3: "El rating es lo fundamental en televisión, la audiencia alega por la baja calidad de los contenidos, pero al final se transmite lo que la gente quiere ver. Si nos enfocáramos en temas culturales no se verían los programas, el público busca el morbo".

Caso 4: "Las marcas de las empresas quieren aparecer y el diario también busca que aparezcan porque eso significa ingresos monetarios. Pero no me importa si una marca me critica por alguna publicación. Al momento de publicar me siento $100 \%$ libre".

Caso 5: "Los medios, en general, se guían por el dinero y el rating, pero en el caso nuestro que somos un canal de cable, no nos interesan esos factores. No nos dejamos influir por los auspiciadores; pero aunque no queramos que lo hagan, a veces hay que ceder y dejar que nos presionen".

Caso 7: "A la empresa le importan las utilidades, al editor le importa publicar la noticia antes $y$, al periodista, la calidad".

\subsection{Presiones Políticas}

Se preguntó a los periodistas si sentían alguna presión de tipo político de parte de los dueños o editores de los medios. Hasta 1989 en Chile existió censura política hacia los medios.

Caso 1 al 8 coinciden en afirmar que no hay presiones políticas.

\subsection{Presiones Personales}

Esta pregunta tiene relación con la supervisión del trabajo de cada reportero de parte de los editores.

Caso 1: "No cedería bajo ningún punto de vista a alguna presión de mi editor si eso significara faltar a mi ética de periodista. Yo no tengo miedo de perder el trabajo".

Caso 2: "Yo tengo mucha autonomía, nadie me revisa las notas. Cuando empecé a trabajar estaba complicada porque me gustaba la radio y quería hacer bien mi trabajo. Yo le hago correcciones a mi editor y a mi directora, con mucha confianza, porque todos queremos conseguir un buen producto".

Caso 3: "Soy totalmente remplazable, de hecho, mis compañeros son mi competencia. Estamos constantemente compitiendo".

Caso 4: "No tranzo con mi ética periodística, pero he estado en situaciones en que me ha tocado hacerlo y lo soluciono omitiendo mi nombre en el artículo".

Caso 5: "En el caso de Internet es muy distinto ya que nosotros no reporteamos sino que somos un reflejo de lo que cubren los reporteros de otros medios. Entonces tenemos la suerte de no vernos influidos por factores externos".

Caso 7: "Hay que ser coherente con las convicciones personales. Eso no lo 
enseñan en la universidad. Si el periodista siente que lo están pasando a llevar, tiene que irse de ese medio".

Caso 8: "Si mi editor me exige y no estoy de acuerdo, no cedo".

\subsection{Presiones de Autoridad}

Esta pregunta se refiere al grado de control que sienten los periodistas de parte de los dueños de las empresas.

Caso 1: "Los editores no son los culpables de la falta de independencia. En Chile no existe una ley o un control sobre quienes pueden comprar medios. Los medios se agrupan en manos de pocos".

- "Existe presión y cierto temor a que no exista una real independencia. Yo estoy segura de que en todo medio los periodistas buscan buenos temas y los editores los respaldan, pero siempre existe la restricción de la gerencia y, mientras eso no sea regulado, la independencia periodística seguirá truncada. Pienso que la independencia no existe".

- "Hay inseguridad de los periodistas cuando se deben cubrir temas que afecten a los dueños, ahí no soy independiente. Los auspiciadores jamás han sido problema, tampoco la reacción del público. Pero sí hay problema cuando existen intereses de poder, no sólo directos... hay que fijarse en quienes son los dueños del canal, de qué partido político son, etc".

Caso 2: "Hay que respetar la línea editorial y a los auspiciadores. Pero me parece que lo que más influye, en todos los medios, es el trato con la gerencia".

- "El medio entrampa al editor y el editor entrampa a los periodistas. La censura viene desde arriba del editor, no es culpa suya cuando una noticia no se publica tal como ha sido escrita".

Caso 3: "Los que mandan son los dueños, la gerencia. Yo llegué cuando se estaba pasando por un cambio de dueño en el medio y ese cambio se notó mucho".

- "Los canales de televisión son empresas y hay que partir de esa base, eso influye mucho. Por eso, los periodistas tienen poca autonomía".

Caso 4: "Mi editor, director y coordinador de editores me escudan completamente. Se promueve la resistencia a presiones, de hecho, ellos le dicen a las mismas empresas auspiciadoras que me dejen tranquilo".

- "He publicado notas porque me lo piden desde la gerencia, porque le deben un favor a alguna empresa. Yo he alzado la voz respecto al tema y he hecho sentir mi molestia".

Caso 5: "Mi editor está constantemente reforzando nuestra seguridad y trabajo, sabe hacer su rol".

- "En mi caso, al trabajar en Internet, no tenemos presión por parte de la gerencia".

- "Es mucha, quizá demasiada, la influencia de la gerencia y dueños del medio. Se cuidan mucho sus intereses".

Caso 6: "Los auspiciadores no tienen influencia alguna".

- "Quienes llevan más años en este trabajo hacen críticas constructivas, que se toman como aprendizaje porque ellos son como verdaderos profesores".

Caso 7: "La mayor presión se la hace uno mismo, se autocensura por temor 
a una reprimenda o al despido. Yo le temo a los editores más que a la gerencia ya que no hay una relación directa con ella. Si la gerencia tiene un problema o no le gustó algo, se lo dirá al equipo de editores, no al periodista".

- "El editor se va a salvar él antes que dar la cara por los periodistas".

Caso 8: "No tengo 100\% de independencia, hay que ser muy cauteloso con los temas de interés de la autoridad".

\subsection{Presiones de la opinión pública y} redes sociales

Como ya se ha dicho, hoy tiene mucha importancia la opinión del público. Se trata de un público participativo y opinante que, incluso, llega a proponer noticias o envía fotos o videos para incluir en los medios.

Caso 1: "El tema de las redes sociales le interesa al medio por las reacciones del público. Los encargados de Internet están constantemente tanteando el pulso a las redes sociales. Se buscan temas también a través de ellas. Pero no nos afecta, o quizás sólo en su justa medida, porque tenemos claro que las redes sociales no abarcan a la mayoría de la población, sino que, por medio de ellas, opina un número muy limitado de personas. La presión probablemente vendrá más adelante, ya que la tecnología avanza a pasos agigantados. Entonces, cuando el público que opine a través de redes sociales constituya un número considerable, a los periodistas se les impondrá una capacidad de redacción mucho más rápida. Los periodistas tendrán que ser aún más asertivos y lograr adelantarse en los temas".
Caso 2: "No estoy segura de si hay verdaderamente una presión generada por las nuevas plataformas, pero sí me doy cuenta de que hay un feedback muy potente. Twitter puede tener gran repercusión. El periodista hoy tiene más cuidado porque está muy expuesto".

Caso 3: "Me influye Twitter, yo me metía para ver las opiniones de mis notas, pero me afectaba, así es que lo dejé de hacer. El público comenta para alabar y para criticar lo publicado. Pienso que Twitter marca pauta, pero hay que confirmar los hechos y lo que se dice".

Caso 4: "Se trata de opiniones, que no son representativas de la sociedad y no creo que sea conveniente darles tanta importancia".

Caso 5: "No se puede dar en el gusto a todos, esa es la premisa para enfrentarse a las redes sociales".

Caso 8: "Lo que más me quita autonomía en el trabajo son las presiones externas por parte de la opinión pública”.

- "Yo creo que muchos editores no se atreven a abordar ciertos temas relevantes para la sociedad por miedo a lo que puedan pensar. Siento que son demasiado cuidadosos".

\section{Conclusiones}

Las investigaciones ya desarrolladas (Yez, Gronemeyer y Mellado) llegaron a conclusiones que coinciden con algunos de los resultados de la presente investigación.

Las tres investigaciones concluyeron de manera importante que los periodistas sienten limitada su autonomía por las metas económicas determina- 
das por la empresa donde trabajan: "la guerra por el rating". La mitad de los periodistas entrevistados en el presente estudio consideró importante dicha presión a la hora de responder sobre cuáles eran los principales problemas que restan autonomía en su trabajo periodístico. Explicaron que muchas veces les gustaría desarrollar otro tipo de noticias de más calidad informativa que supuestamente otorgarían menos $\mathrm{ra}$ ting al canal, y no pueden hacerlo.

En cuanto a la influencia de los auspiciadores de los medios sobre la información, la mayoría de los periodistas entrevistados plantearon que no existe presión alguna por parte de éstos. Sólo un entrevistado, que trabaja en un medio que trata en su mayoría temas económicos, explicó que la influencia para él era muy alta.

Por otra parte, en el ámbito político todos los periodistas encuestados estuvieron de acuerdo con un mismo planteamiento: no hay presión política. Incluso, todos ellos explicaron que existía un gran respeto por las distintas posiciones existentes dentro del medio. Dicho planteamiento deja en evidencia que la visión de investigaciones pasadas acerca de la influencia de la censura del periodo de gobierno militar actualmente ya no existe.

Cabe mencionar que, si bien a los periodistas no se les presionaba bajo ningún punto en su postura política, ellos admitieron tener que desarrollar con especial cuidado las noticias que tuviesen que ver con los intereses políticos de la gerencia del medio en el cual trabajan.
Otro factor que permite concluir que ha desaparecido la influencia del gobierno militar, es que todos los entrevistados aclararon que, por ningún motivo, cederían ante presiones que se opusieran con su ética profesional. Todos, a excepción de uno, señalaron que no tolerarían una censura explícita, indicando que prefieren renunciar.

Otra conclusión que refleja cierta inseguridad por parte de los periodistas es que todos ellos se consideran absolutamente remplazables y prescindibles, incluso admitiendo cierto espíritu de competencia con compañeros.

Las investigaciones previas analizadas en este trabajo, destacan el importante papel que tiene el editor en la sala de prensa. Su buen desempeño, actitud y relación con los periodistas es fundamental. Al entrevistar a los ocho periodistas, se reafirma dicha hipótesis, ya que coincidió que quienes se sienten protegidos por su editor (4/8), tienen buena relación con él y lo consideran clave para lograr un buen trabajo, defienden la idea de que no son ellos los responsables de la falta de autonomía e independencia de los periodistas chilenos.

Por otra parte, las redes sociales, en especial Twitter, no se consideran un factor relevante en la falta de autonomía del periodista. Si bien hubo un par de casos que indicó que existía una influencia y les afectaba ser criticados por la red social, la mayoría consideró que Twitter no representa a la gran sociedad chilena. Pero si bien no le dan tanta relevancia a las redes sociales, admiten que hoy ellos son más cuidadosos con 
sus notas debido a que están más expuestos a críticas.

Otra conclusión que se puede sacar de las entrevistas es que los ocho periodistas reconocieron que existía un problema con la autonomía de los comunicadores en relación a los dueños de la empresa periodística. Esta conclusión también fue muy relevante en las investigaciones porevias mencionadas. Todos ellos apuntaron como principal factor influyente en la independencia a la gerencia de los medios de comunicación. Indicaron que, aunque un periodista tenga sus principios, haga un buen trabajo y su editor lo apruebe, los dueños de los medios son quienes finalmente deciden qué pasa con su noticia. Los entrevistados estuvieron de acuerdo en que si el material periodístico interfería con los intereses de los altos mandos, no saldría a la luz.

Se puede afirmar, después del análisis de las entrevistas, que no hay una correlación directa entre el tipo de medio (diario, televisión, radio, revista e Internet) y las percepciones de autonomía de los periodistas. Las respuestas sobre las relaciones con los dueños de la empresa, auspiciadores, editores y público son independientes del medio en el que trabajan los periodistas entrevistados.

\section{Referencias Bibliográficas}

Aznar, H. (2004). Nuevos códigos de ética y nuevas formas de entender el periodismo. Revista Latina de Comunicación Social, 58, pp. 45-61.

Barrios, M. y Arroyave, J. (2007). Perfil Sociológico de la profesión del periodista en Colombia: Diálogo íntimo con el ser humano detrás de las noticias. Revista Diálogos en Comunicación, 75, p. 1-26.

Blank, G., Dutton, W. y Newman, N. (2012). Social media in the changing ecology of news: The fourth and fifth estates in Britain. International Journal of Internet Science, 7 (1), 6-22.

Blank, G. (2013). Who creates content? Information, Communication \& Society, 16(4), 590-612. Campos Freire, F. (2008). Las redes sociales trastocan los modelos de los medios de comunicación tradicionales. Revista Latina de Comunicación Social, 63, 287-293.

Canales, M. (2006). Metodologías de investigación social. Santiago: Lom.

Dunham, J., Nelson, B. y Aghekyan, E. (eds) (2015). Freedom of the Press in 2014. New York: Freedom House. Recuperado de http://freedomhouse.org/sites/default/files/ FreedomofthePress_2015_FINAL.pdf

Domingo, D. et al. (2007). Four dimensions of journalistic convergence: A preliminary approach to current media trends at Spain. Paper presented at the $8^{\text {th }}$ International Symposium on Online Journalism, Austin, Texas. Rescatado del URL: http:// online.journalism.utexas. edu/2007/papers/Domingo.pdf

Dutton, W. H. \& Blank, G. (2013). The Emergence of Next-Generation Internet Users. In $A$ Companion to New Media Dynamics, pp. 122-141.

Flores, J.M. (2009). Nuevos modelos de comunicación, perfiles y tendencias en las redes sociales. Comunicar, 33, 73-81. 
Giles, R. (1993). Newsroom Management: A Guide to Theory and Practice. Detroit: Media Management Books.

Greene, F. (2012). La objetividad como rutina periodística. Pamplona: Eunsa.

Greene, F. (2014). Los medios bajo la influencia de las plataformas tecnológicas: ¿en qué medida estas afectan en la toma de decisiones de los editores chilenos? Estudios del Mensaje Periodístico, 20 (2), 64-76.

Gronemeyer, M. E. (2002). Periodistas chilenos: el reto de formar profesionales autónomos e independientes. Cuadernos de Información, 25, 12-22. Recuperado de: http://comunicaciones.uc.cl/prontus_fcom/site/artic/20050402/pags/200504021 45237.html

Guerrero, M. y Márquez, M. (2014). Media Systems and Communication Policies in Latin America. Nueva York: Palgrave Macmillan.

Gutiérrez, A. y Torres, A. (2014). Rutinas periodísticas y autocensura: el ejercicio periodístico en el entorno violento del noreste de México. En 4to. Congreso Nacional de Ciencias Sociales. La Construcción del Futuro. Los Retos de las Ciencias Sociales. San Cristóbal de las Casas, Chiapas.

Hallin, D. y Mancini, P. (2004). Comparing Media Systems: Three Models of Media and Politics. Nueva York: Cambridge University Press.

Hanitzsch, T. (2011). Populist disseminators, detached watchdogs, critical change agents and opportunist facilitators: Professional milieus, the journalistic field and autonomy in 18 Countries. International Communication Gazette, 73(6): 477-494.

Hanitzsch T. y Mellado, C. (2011). What shapes the news around the world? How journalists in 18 countries perceive influences on their work. International Journal of Press Politics, 16(3), 404- 426.

Hanitzsch, T., Mellado, C., Hanusch, F. y Wang Yuen, E.. (2011). Mapping Journalism Cultures Across Nations. Journalism Studies 12 (3), 273- 29.

Hernández, R. (2003). Metodología de la investigación. Ciudad de México: Mc Graw Hill.

Lecaros, M. J. (2004). Hacia un periodismo de calidad. Cuadernos de Información, 27, 34-42. Recuperado de http:/comunicaciones.uc.cl/prontus_fcom/site/artic/20041213/ pags/200412131 64912.html

Lecaros, M. J. (2009). La ética de los profesionales de la información en el seno de las organizaciones informativas. En Egúsquiza, I. (Ed.), La libertad de las conciencias en la regulación del derecho a la información, 55-68. Valencia: Fundación COSO.

McDevitt, M. (2003). In Defense of Autonomy: A Critique of the Public Journalism Critique. Journal of Communication, 53: 155-160.

McQuail, D. (1992). Media Performance: Mass Communication and the Public Interest. New York: Sage Publications.

Mair, P. (2011). Democracies. In: Caramani D (ed.). Comparative Politics (pp.84-102). England: Oxford University Press.

Márquez-Ramírez, M. (2012). Valores normativos y prácticas de reporteo en tensión: Percepciones profesionales de periodistas en México. Cuadernos de Información, 30, $97-110$. 
Mellado, C. y Humanes, M. L. (2012). Modeling perceived professional autonomy in Chilean journalism. Journalism 13(8), 985-1003.

Mellado, C. (2011). Examining professional and academic culture in chilean journalism and mass communication education. Journalism Studies, 12 (3), 375 - 391.

Metzler, K. (1986). Newsgathering. New York: Prentice-Hall.

Muñoz, M. (2004). La autorregulación como espacio de libertad informativa. Paradigma Comunicacional Comercial, 33, 124-135. Recuperado de http://biblioteca.universia.net/ html_bura/ficha/params/title/autorregulacion

Nygren, G. (2012). Journalism in Russia, Poland and Sweden: Traditions, Cultures and Research. Stockholm: Södertörn University Press.

Orihuela, J. L. (2008). Internet: la hora de las redes sociales. Nueva Revista de Política, Cultura y Arte, 119, $57-62$.

Ortega, F. y Humanes, M. (2000). Algo más que periodistas. Sociología de una profesión. Barcelona: Ariel.

Puente, S., Edwards, C., Delpiano, M. O. (2014). Modelamiento de los aspectos intervinientes en el proceso de pauta periodística. Palabra Clave, 17 (1), 186-208.

Reich, Z. y Hanitzsch, T. (2013). Determinants of journalists' professional autonomy: Individual and national level factors matter more than organizational ones. Mass Communication and Society, 16(1), 133-156.

Ruiz Olabuénaga, J. I. (2003). Metodología de la investigación cualitativa. Bilbao: Universidad de Deusto.

Sánchez Tabernero, A. (2000). Dirección Estratégica de Empresas de Comunicación. Madrid: Cátedra.

Sancho, F. (2004). En el corazón del periódico. Pamplona: Eunsa.

Schudson, M. (2005). Autonomy from what? En: Benson R, Neveu E (eds). Bourdieu and the Journalistic Field (pp. 214-223). Malden, MA: Polity Press.

Sjovaag, H. (2013). Journalistic autonomy; between structure, agency and institution.

Skovsgaard, M. (2014). A tabloid mind? Professional values and organizational pressures as explanations of tabloid journalism. Media Culture \& Society, 36 (2), 200-218.

Nordicom Review: Nordic Research on Media and Communication, 34, 155-167.

Taufic, C. (2005). La autorregulación del periodismo. Manual de ética periodística comparada, 20, 178-189. Recuperado de http://www.c3fes.net/docs/autorregulacion.pdf.

Vieytes, R. (2004). El problema de investigación. Metodología de la Investigación en Organizaciones, Mercado y Sociedad. Epistemología y técnicas, 14, 127-139.

Weaver, D., Beam R. y Brownlee B. (2006). The American Journalist in the 21st Century: US News People at the Dawn of a New Millennium. New York: Routledge.

Weaver, D. y Willant, L. (2012). The Global Journalist in the 21st Century. New York: Routledge. Yez, L. (2011). Estado actual y desafíos profesionales de los periodistas chilenos. Cuadernos de Información, 34, pp. 14-21. 